\title{
3-D OFFLINE SigNATURE VERIFICATION WITH CONVOlUtional NeURAL NETWORK
}

\author{
Na Tyrer ${ }^{1}$, Fan Yang ${ }^{1}$, Gary C. Barber ${ }^{1}$, Guangzhi Qu ${ }^{1}$, \\ Bo Pang ${ }^{1}$ and Bingxu Wang ${ }^{1,2}$ \\ ${ }^{1}$ Automotive Tribology Center, Department of Mechanical Engineering, School \\ of Engineering and Computer Science, Oakland University, \\ Rochester, Michigan, 48309, USA \\ ${ }^{2}$ Faculty of Mechanical Engineering and Automation, Zhejiang Sci-Tech \\ University, Hangzhou, Zhejiang, 310018, P.R.China
}

\begin{abstract}
Signature verification is essential to prevent the forgery of documents in financial, commercial, and legal settings. There are many researchers have focused on this topic, however, utilizing the 3-D information presented by a signature using a 3D optical profilometer is a relatively new idea, and the convolutional neural network is a powerful tool for image recognition. The present research focused on using the 3 dimensions of offline signatures in combination with a convolutional neural network to verify signatures. It was found that the accuracy of the data for offline signature verification was over 90\%, which shows promise for this method as a novel method in signature verification.
\end{abstract}

\section{KEYWORDS}

Signature Verification, 3D Optical Profilometer, Convolutional Neural Network

\section{INTRODUCTION}

With the security requirements related to signing contracts, signing checks, etc, handwriting has become a more and more important factor in current society. An individual's signature is easily influenced by emotions and may vary day-to-day or can be completely different over time. Signature fraud is difficult for a human's eye to identify; thus it is important to find an automatic signature verification method. To prevent signature fraud, the handwriting verification system becomes crucial. Handwriting verification is widely used in many fields, it is essential to prevent the forgery of documents in financial, commercial, and legal environments.

Signature verification can be classified into two types: online and offline. For the online type, a special pen and an electronic surface are required. The stored data provides information such as the pen's position, velocity, acceleration, pressure, and angle as a function of time. However, using an electronic pen can be very different from using a pen. For the offline type, only the signed documents are available to be analyzed. While online signature verification provides more information, it needs a specific system that is not always available. What's more, most of the important documents require a handwritten signature. Therefore, exploring improved methods to verify offline signatures is essential.

Several studies have been conducted on this topic. In "Automatic Signature Verification", the author presented the state of the art in automatic signature verification. It contained a David C. Wyld et al. (Eds): ACITY, DPPR, VLSI, WeST, DSA, CNDC, IoTE, AIAA, NLPTA - 2020 pp. 221-228, 2020. CS \& IT - CSCP 2020

DOI: $10.5121 /$ csit.2020.101518 
comprehensive bibliography of more than 300 selected references as an aid for researchers working in the field including both online and offline signature analysis [1]. The researchers summarized two type of features - function features and parameter features. Function features are position, velocity, acceleration, pressure, force, direction, etc. Parameter features are global parameters such as, number of penups or pendowns, time duration of positive, etc, and local parameters such as component priented and pixel oriented. It was concluded that based on current methods, the accuracy is very promising. It also pointed out that the research should be more focused on device interoperability and the need for specific investigations. For the online signature verification, the speed of the pen is used as a dynamic feature of the signature [2]. The pen-input on-line signature verification incorporating pen-position, pen-pressure, and peninclinations trajectories was developed in "On-line Pen Input Signature Verifier PPI (penPosition/ pen-Pressure/pen-Inclination)" [3].

For the offline signature verification, an offline signature verification system using a convolutional neural network was developed [4]. The resulting model was based on pre-trained VGG16 architecture using ICDAR 2011 SigComp dataset to train with transfer learning. J. Coetzer et al. [5] developed an off-line signature verification system that uses features that are based on the calculation of the Radon transform (RT) of a signature image. Each writer's signature is subsequently represented by a hidden Markov model (HMM). In "A smoothness index-based approach for offline signature verification" a method was developed based on a smoothness criterion [6]. Although skilled forgery signatures are very similar to genuine, they are generally less smooth and natural on a detailed scale than the genuine ones. R. Sabourin et al. [7] investigated the use of shape matrices as a mixed shape factor for off-line Signature Verification. Originally, shape matrices have been used for planar shapes, however, the reseachers used shape matrices to compare signatures. They concluded that shape factor is relatively well suited for the global interpretation of signature images. Luiz G. Hafemann et al [8] performed extensive experiments on four deep convolutional neural networks databases which are GPDS, MCYT, CEDAR and Brazilian PUC-PR datasets. The results show large improvement on GPDS-160 database. As shown above, the challenge of offline signature is not only extracting the signature but also to choose a reliable database.

Several research papers considered online signature pen pressure analysis, which requies an electronic pen. However, there is a difference between writing with a digital pen versus writing with an actual pen. With a 3D optical profilometer, offline signature pen pressure analysis can be determined precisely. Previous research has focused on 2D information for the offline signatures, which for highly skilled forgery may appear highly similar to the actual signature, but the pen pressure is harder to mimic. The purpose of this research is to determine the $3 \mathrm{D}$ information of offline signatures and utilize a convolutional neural network to allow a computer to extract features of the signature.

\section{Experimental Procedure}

The present research consisted of two tasks. One is planning to explore deep learning algorithms, i.e. convolutional neural network which is used to train and test the images for 3-D information. Experiments were conducted to test the network by using the signatures during training. The second task was to utilize a Bruker Contour GT-K 3D Optical Profilometer to extract the offline signature. Figure 1 shows a 3-dimensional image letter 'a' with various depths that occur due to indentations in the paper when the letter was written. For example, the bright blue color represents areas where pen pressure was high producing relatively deeper indentations, the green color represents relatively low pen pressure and the red color represents the original surface of the paper. 


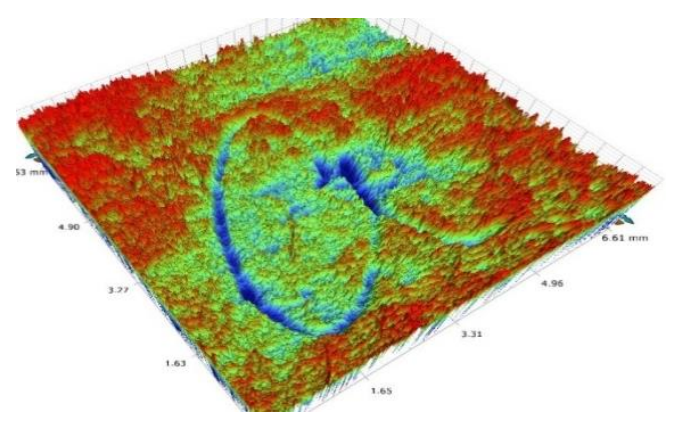

Figure 1. The scanned 3-D image of the handwritten letter ' $a$ '

Figure 2 shows the typical convolutional neural network $(\mathrm{CNN})$ architecture. Regular neural networks take the input data and convert it into a one-dimensional vector of neurons, while CNN's work in 3 dimensions: width, height, depth. For example, the input is the image. The width and the height are dimensions of the image, and the depth stands for 3 channels (Red, Green, and Blue). Figure 3 shows the neuron in 3 dimensions as visualized in one of the layers. In addition, the neurons in a layer will only be connected to a small region of the layer before it, rather than all of the neurons in a fully connected position. At the end of the CNN architecture, the full image was reduced into a single vector of class scores, arranged along the depth dimension.

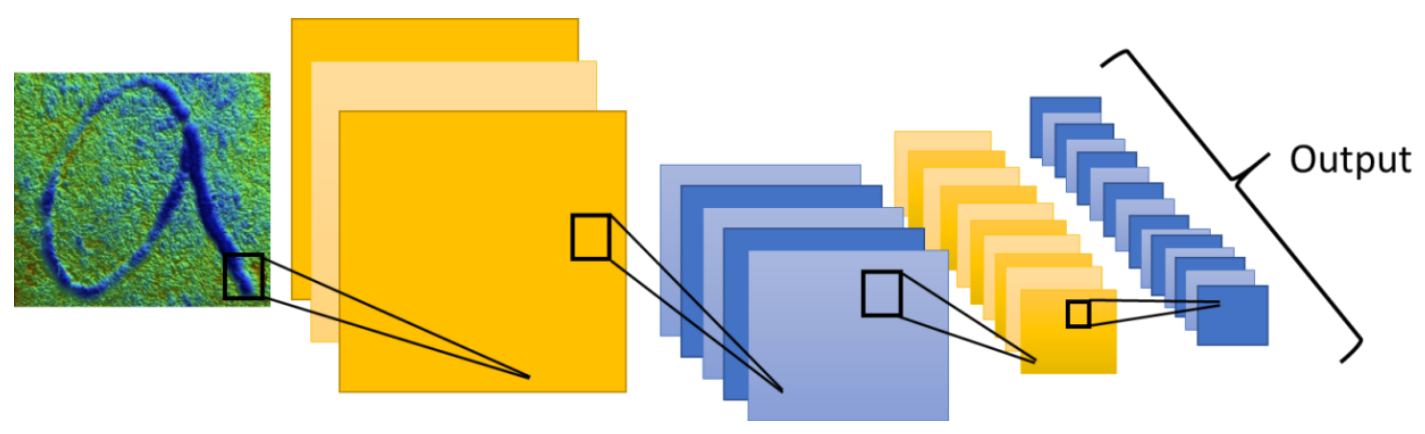

Figure 2. Typical CNN architecture

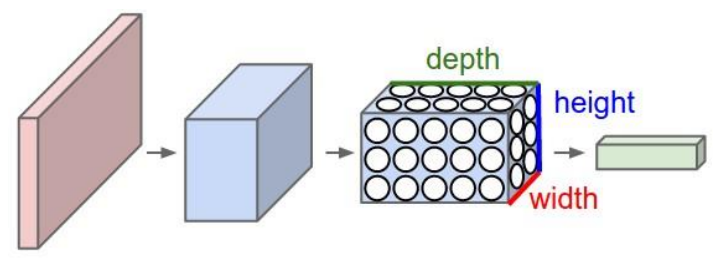

Figure 3. The neuron in 3D as visualized in one layer [9]

The advantage of this technique is to analyze the pen contact pressure on surfaces such as paper, instead of an electronic pen. As shown in Figure 4(a)-(d), there are two participants who wrote "Tom" and "Jim" on a piece of paper. They may not show significant differences by the naked eye. However, using pen pressure as shown in Figure 4(e)-(h) a significant difference can be observed. Using this technique combined with the current signature recognition technique, the accuracy of signature recognition is expected to be enhanced. 


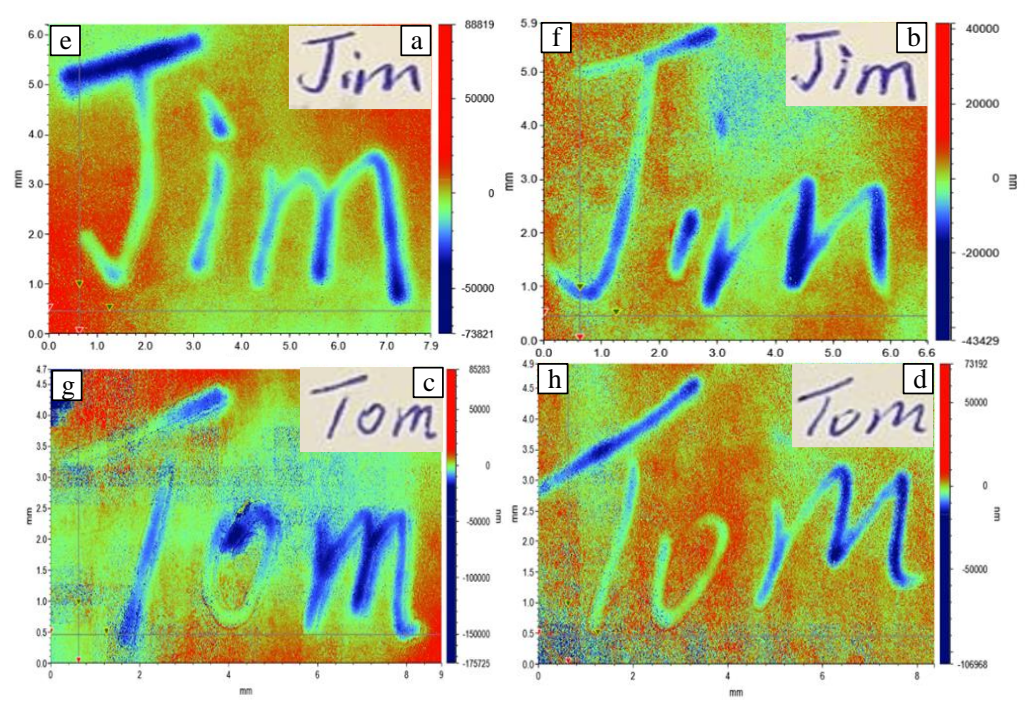

Figure 4. (a) (c) (e) (g) “Jim" “Tom" wrote by Participant One (b) (d) (f) (h) "Jim" “Tom" wrote by Participant Two

To demonstrate the concept of this research, a simply database was created. The database is composed of 80 offline samples written by two participants. Because of the time constraints of the 3-D machine scanning process, the letter ' $\mathrm{a}$ ' is used to demonstrate the concept. In the experiment, every participant wrote 40 letters ( 35 for training and 5 for testing). Considering the instability which occurs in the process of collecting data, the "signature "is recorded on the same sheet of paper using the same pen to eliminate potential variables.

The optical profilometer has different types of filters to help eliminate noise, remove the tilt of the surface, etc. As shown in Figure 5, it can measure the various depths of the surface in 2D and 3D mode.
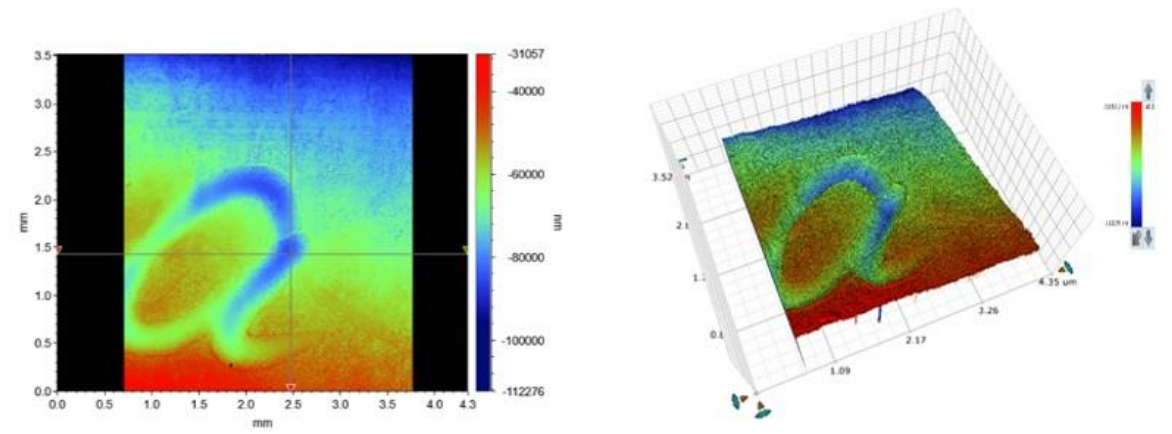

Figure 5. Same handwriting with different mode (left - 2D image, right $-3 \mathrm{D}$ image)

\section{Results}

In the experiments, the database (35 letters of each participant) was divided into a training set (Figure 6) and a testing set in a ratio of 3:7 to train the model, and 5 unseen data were used to test the performance. Five of the thirty-five training letters after the scanning process are shown below: 

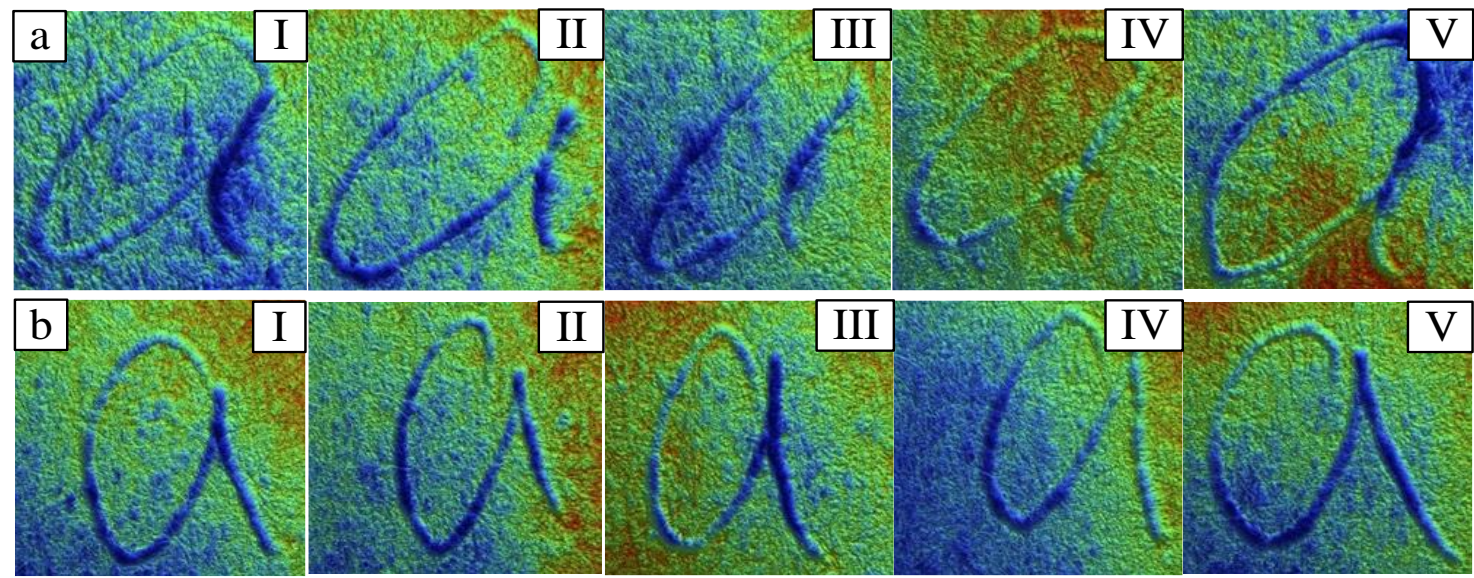

Figure 6. (a) Five training data from Participant One (b) Five training data from Participant Two

For the two participants, five handwritten letters were used to test the accuracy of the model. The data are shown in Figure 7.

For participant one, the 5 data were used to test the model ten times. Then an average accuracy was calculated which was over $90 \%$. Three of the ten results are shown below:

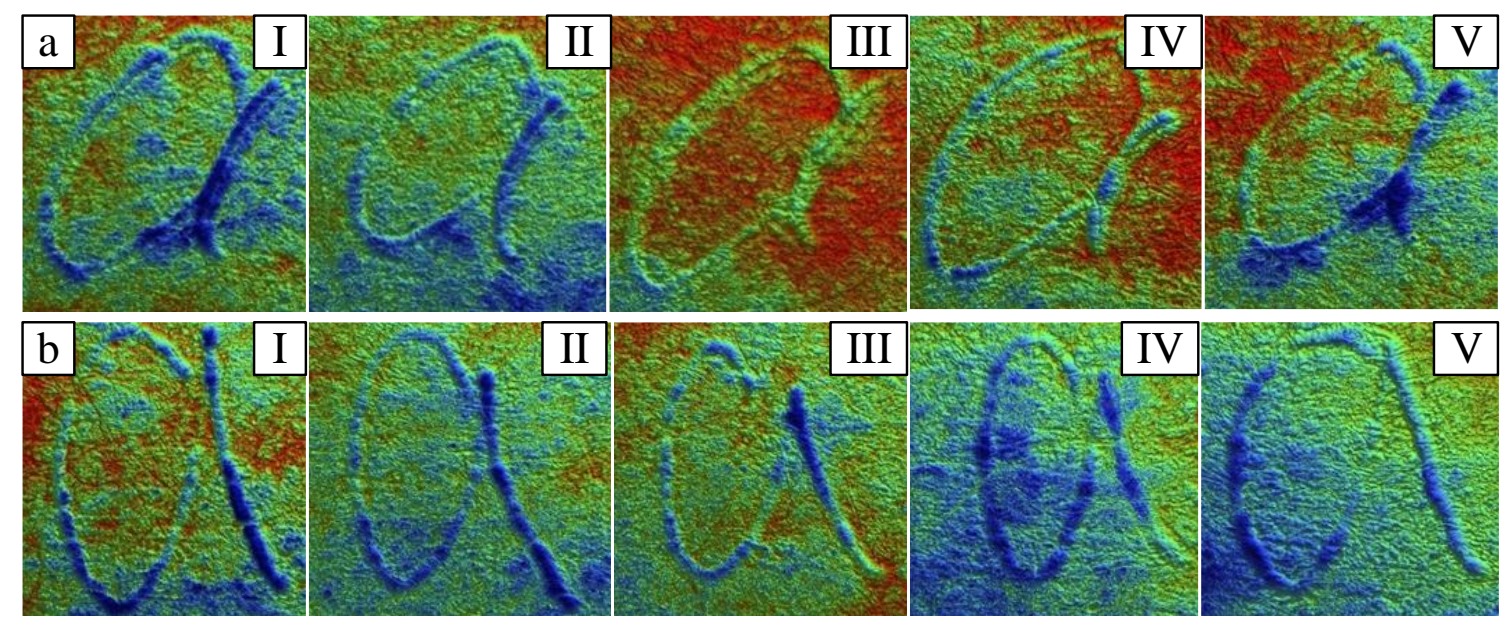

Figure 7. Five handwritten letters for the accuracy tests of the model (a) Participant One (a) Participant Two 


\begin{tabular}{|c|c|c|}
\hline \multirow{3}{*}{ Participant One } & $1^{\mathrm{st}}$ & 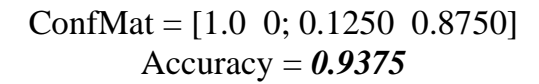 \\
\hline & $2^{\text {nd }}$ & $\begin{array}{c}\text { ConfMat }=\left[\begin{array}{lll}0.9583 & 0.0417 ; 0 & 1.000\end{array}\right] \\
\text { Accuracy }=\mathbf{0 . 9 7 9 2}\end{array}$ \\
\hline & $3^{\text {rd }}$ & 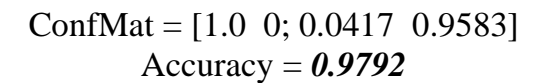 \\
\hline
\end{tabular}

For participant two, the same method applied to participant one, three of the ten test results are shown below.

Table 2. Summary results of Participant Two

\begin{tabular}{|c|c|c|}
\hline \multirow{3}{*}{ Participant Two } & $1^{\text {st }}$ & $\begin{array}{l}\text { ConfMat }=\left[\begin{array}{lll}1.0 & 0 ; 0.0417 & 0.9583\end{array}\right] \\
\text { Accuracy }=\mathbf{0 . 9 7 9 2}\end{array}$ \\
\hline & $2^{\text {nd }}$ & $\begin{array}{l}\text { ConfMat }=\left[\begin{array}{lll}0.9167 & 0.0833 ; 0 & 1.000\end{array}\right] \\
\text { Accuracy }=\mathbf{0 . 9 5 8 3}\end{array}$ \\
\hline & $3^{\mathrm{rd}}$ & $\begin{array}{l}\text { ConfMat }=\left[\begin{array}{lll}0.9583 & 0.0417 ; 0 & 1.000\end{array}\right] \\
\text { Accuracy }=\mathbf{0 . 9 7 9 2}\end{array}$ \\
\hline
\end{tabular}

\section{Conclusion}

As the results have shown, with the combination of the optical profilometer and the CNN, the accuracy of the data for offline signature verification is very promising. For the 3D signature verification method, a new option is provided to verify signatures and achieve a high accuracy in the analysis of offline handwriting on top of other researchers have achieved In future research, the training database needs to be developed with much larger data inputs as training sets by collecting more offline signatures from various people. The more input data collected, the more accurate the results will be. In addition, a more complicated algorithm to combine all relevant factors, such as handwriting similarity, pen-pressure, and pen-inclination trajectories, etc should be developed. Using the optical profilometer is time-consuming but introducing one more factor is a significant contribution for offline analysis. Even with different types of paper, the relative pen pressure has value for fraud detection. 


\section{REFERENCES}

[1] D, Impedovo \& G, Pirlo, (2006) “Automatic Signature Verification: The State of the Art”, IEEE Transactions on Systems, Man, and Cybernetics-Part C: Applications and Reviews, Vol. 38, No. 5, pp609.

[2] A, Al-Shoshan, (2008) "Handwritten Signature Verification Using Image Invariant and Dynamic Features", Proceedings of the International Conference on Computer Graphics, Imaging and Visualization (CGIV 2006), Jul 2006, Sydney (Australia). pp173-176.

[3] Y, Komiya \& T, Matsumoto, (1999) "On-line Pen Input Signature Verifier PPI (pen-Position/ penPressure/pen-Inclination)”, IEEE Smc'99 Conference Proceedings. 1999 IEEE International Conference on Systems, Man \& Cybernetics, Oct 1999, Tokyo (Japan).

[4] G. Alvarez, B. Sheffer, M. Bryant, Offline Signature Verification with Convolutional Neural Networks

[5] J, Coetzer, B, Herbst \& J. Preez, (2006) "Off-Line Signature Verification: A Comparison between Human and Machine Performance", Tenth International Workshop on Frontiers in Handwriting Recognition, Oct 2006, La Baule (France).

[6] B, Fang, Y, Wang, C, Leung, P, Kwok, K, Tse, Y, Tang, \& Y, Wong, (1999) “A Smoothness IndexBased Approach for Offline Signature Verification", Proceedings of the Fifth International Conference on Document Analysis and Recognition ICDAR'99, Sep 1999, Banalore (India). pp785791.

[7] R, Sabourin, J, Drouhard \& E, Wah, (1997) "Shape Matrices as A Mixed Shape Factor for Offline Signature Verification", Proceedings of the Fourth International Conference on Document Analysis and Recognition ICDAR'97, Aug 1997, Ulm (Germany). Vol. 2, pp661-665.

[8] Luiz G.Hafemann, Robert Sabourin, Luiz S.Oliveira, (2017) "Learning Feautres For Offline Handwritten Signaature Verification Using Deep Convolutional Neural Networks", Pattern Recognition 70 (2017) pp 163-176

[9] CS231n Convolutional Neural Networks for Visual Recognition, http://cs231n.github.io/convolutional-networks/

\section{Authors}

NA TYRER is currently a Ph.D. student at Automotive Tribology Center at Oakland University. She is closely working with Automotive OEMs as a research assistant to investigate the tribological performance of automotive parts. Her main research field is the tribological behavior of electrical coatings.

FAN YANG received her B.S. degree from the Beijing Information Science and Technology University and M.S. degree from the Oakland University in 2020.

GARY C. BARBER received his PhD from the University of Michigan in 1987. He is currently a Professor and Director of the Automotive Tribology Center at Oakland University in Rochester, Michigan. Dr. Barber is a fellow of the Society of Tribologists and Lubrication Engineers (STLE) and has published more than 125 papers in journals and conference proceedings. His research areas include the effects of various heat treatments on the tribological behavior of steels and cast irons and the use of nanofluids as lubricants.

GUANGZHI QU received the B.E. and M.E. degrees from the Department of Computer Science and Engineering, Beihang University, and the Ph.D. degree from the University of Arizona in 2005. He is currently a professor at Oakland University in Rochester, Michigan. His research areas include the data mining, machine learning, operating systems, and program analysis.
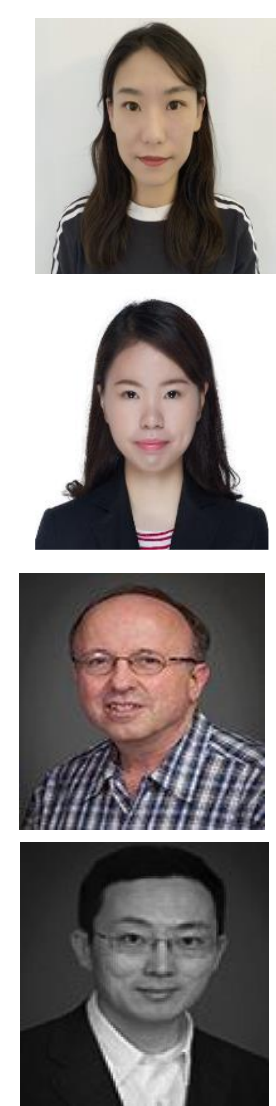
BINGXU WANG received the Ph.D degree from Oakland University, Michigan, USA, in 2018. He is currently an assistant professor of the Faculty of Mechanical Engineering and Automation at Zhejiang Sci-Tech University, China. Dr. Wang is a member of the Society of Tribologists and Lubrication Engineers (STLE) and the Society of Automotive Engineers (SAE), and has published about 28 papers in various peer reviewed journals and conference proceedings. His research areas include 3D surface measurements, metallurgical evaluation, austempered ductile iron, heat treatment design, tribological properties and nanofluids applications.

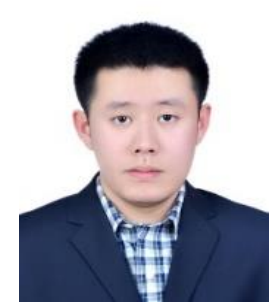

(C) 2020 By AIRCC Publishing Corporation. This article is published under the Creative Commons Attribution (CC BY) license. 